\title{
Scattering matrix approach to interacting electron transport
}

\author{
D. Oehri, ${ }^{1}$ A.V. Lebedev, ${ }^{1}$ G.B. Lesovik, ${ }^{2}$ and G. Blatter ${ }^{1}$ \\ ${ }^{1}$ Theoretische Physik, Wolfgang-Pauli-Strasse 27, ETH Zurich, CH-8093 Zürich, Switzerland \\ ${ }^{2}$ L.D. Landau Institute for Theoretical Physics RAS, 117940 Moscow, Russia
}

(Dated: May 23, 2018)

\begin{abstract}
We investigate the modification in mesoscopic electronic transport due to electron-electron interactions making use of scattering states. We demonstrate that for a specific (finite range) interaction kernel, the knowledge of the scattering matrix is sufficient to take interaction effects into account. We calculate perturbatively the corrections to the current and current-current correlator; in agreement with previous work, we find that, in linear response, interaction effects can be accounted for by an effective (renormalized) transmission probability. Beyond linear response, simple renormalization of scattering coefficients is not sufficient to describe the current-current correlator, as additional corrections arise due to irreducible two-particle processes. Furthermore, we find that the correlations between opposite-spin currents induced by interaction are enhanced for an asymmetric scatterer, generating a nonzero result already to lowest order in the interaction.
\end{abstract}

PACS numbers: 73.23.-b, 03.65.Nk, 72.70.+m, 73.50.Bk

\section{INTRODUCTION}

Transport through mesoscopic systems in the presence of particle scattering and interaction has been at the focus of experimental and theoretical studies during several decades. The scattering matrix theory reducing the problem to asymptotic states ${ }^{1 / 3}$ proved to be an efficient tool characterizing transport in the presence of singleparticle scattering and for non-interacting systems. Beyond calculation of the current, the scattering matrix formalism was used $\frac{\sqrt{45}}{15}$ to determine the noise properties of devices and provided the complete probability distribution of transmitted charge through the calculation of the full counting statistics (FCS $)^{6 / 7}$. Several reviews dealing with the scattering matrix approach to electron transport are available today 89 .

Effects of electron-electron interactions give rise to numerous interesting phenomena, e.g., Coulomb blockade $e^{10}$, Kondo effect $\frac{10}{10}$, or the 0.7 anomaly 11 in transport, or entanglement and decoherence within the context of quantum processing in mesoscopic systems ${ }^{12}$. Interactions arise locally and thus involve the particle wave function within the domain where interactions take place. A natural way to tackle the combined problem of scattering and interactions is to start with the interacting problem (e.g., the quantum dot) and include scattering thereafter, as in the tunneling approach which treats the scattering part perturbatively. In the present paper, we take an alternative approach which describes the scattering part exactly for any scatterer. We then have to compromise with the interaction, for which we choose a particular kernel which is constant over the scattering region. This scheme allows us to express the full interacting Hamiltonian through the exact scattering coefficients of the non-interacting system. The approach can be extended to the case of electron-photon/plasmon and electron-phonon interactions, where the wave function of the scattered low-energy mode (rather than the twoparticle interaction kernel) is approximately constant over the region of scattering and interaction. This idea has been exploited before in the study of photon/plasmon generation by electron scattering, as discussed in Refs. $13-15$.

Accounting for interaction effects in electronic transport is a highly non-trivial task and general results are sparse, with notable exceptions such as the generalized Landauer formula due to Meir and Wingreen 16 , however. Still, considerable progress can be made for specific systems: Studies of the (Anderson ${ }^{17}$ ) impurity model for quantum dots provide non-perturbative results for the non-linear $I-V$ characteristics $\frac{18}{}$, for the shot noise $e^{19}$, and perturbative results (and beyond) are available for the full counting statistics 20 . The same quantities, currentvoltage characteristic ${ }^{2122}$, shot-noise ${ }^{23}$, and full counting statistics $^{24}$, have been found for the interacting resonant level mode $25 \mid 26$. The more general case of a quantum point contact with an arbitrary two-particle interaction has been analyzed with the help of field-theoretic methods: the current and noise have been determined by Golubev et al. ${ }^{27}$ within a saddle-point approximation and the renormalization group flow of the transmission coefficients has been found by Kindermann and Nazarov $\sqrt{28}$ in their study of weak interaction effects on full counting statistics. In our approach, we express the interaction through the non-interacting scattering coefficients of an arbitrary mesoscopic conductor, yet restricted to a capacitive type of Coulomb interaction. Providing this starting point, this interacting Hamiltonian can be used in a simple perturbative calculation as done below, but could also be fed to a more sophisticated technique as those developed in Refs. $27 / 28$.

Much interest has been devoted to the question whether the Landauer formula for the current survives in the presence of interactions, a question that our approach is quite suitable to address. For systems where the interaction among electrons is restricted to a finite region of space, Meir and Wingreen ${ }^{16}$ developed a generalization of the Landauer formula for the current, which was recently 
shown to reduce to the form of the Landauer formula via introduction of an effective transmission 29 . Furthermore, the analysis has been extended to include effects of interactions in the leads 30 . Gogolin and Komnik 20 , devising a method providing the generating function of the full counting statistics, have found that, within linear response, effects of interaction in the Anderson model can be accounted for with a renormalization of scattering coefficients. However, it is still under debate whether all effects of interaction can be included via an effective transmission probability in the Landauer formula 31 .

Below, we consider two (semi-infinite) non-interacting leads connected via a finite region of space where singleelectron scattering and interaction between electrons takes place. We focus on an interaction between electrons which is restricted to a finite region in space and furthermore constant in the region of single-electron scattering. In this case, the matrix elements in the interaction Hamiltonian can be calculated from the knowledge of the asymptotic behavior of the single-particle wavefunctions (scattering matrix). Our study is based on the Keldysh formalism ${ }^{32}$ and includes the non-equilibrium situation with a finite applied bias voltage $V$. We treat the scattering problem exactly by writing the initial density matrix $\hat{\rho}\left(t_{0}\right)$ (with $t_{0} \rightarrow-\infty$ ) in terms of the scattering states of the non-interacting system. Assuming an arbitrary scatterer, we limit ourselves to a perturbative expansion in the interaction and stay with the lowest order (in which case the introduction of Green's function as done below is not a must). Adopting a specific scatterer, e.g., a single resonance level, we push the perturbative expansion to higher order with a selected choice of diagrams.

In a first application of our approach, we determine the interaction-induced correction to transport and noise: to lowest order in the interaction strength $U_{0}$ and to all orders in the applied bias $V$, the corrections to the singleparticle Green's function $G$ and the transport current $j$ can be described through renormalized scattering amplitudes. On the other hand, the corrections to the twoparticle Green's function and the current-noise cannot be cast into the form of renormalized scattering coefficients in general. This is due to the presence of irreducible two-particle processes (vertex corrections). Calculating the current and noise from the single- and two-particle Green's function, we find that in the linear voltage regime $V$ all the effect of interaction can be accounted for by an effective transmission (to order $U_{0}$ ), in agreement with previous findings by Gogolin and Komnik ${ }^{20}$. In the nonlinear voltage regime, this is not the case any more and interaction effects beyond a simple renormalization of the scattering properties appear. Furthermore, it turns out, that the corrections to the two-particle Green's function generate a correlation between opposite-spin currents in an asymmetric device which appears to order $U_{0} V^{2}$, while in a symmetric system ${ }^{20}$ such a correlation arises only in order $U_{0}^{2} V^{2}$. Again, our approach turns out to be quite suitable in unravelling such additional correlations due to an asymmetry of the scatterer, a question that has not been addressed before.

The paper is organized as follows: In Sec. II we introduce the Hamiltonian, briefly explain the nonequilibrium formalism used in the paper, and demonstrate how the interaction matrix elements simplify due to our particular choice of the interaction kernel. In Sec. III, we calculate the lowest order (in interaction) correction to the current and noise correlator and find the interaction-induced correlation between oppositespin currents. We then evaluate the results for the specific case of a single resonance level in Sec. IV including a resummation of diagrams providing the mean-field correction to the single-particle Green's function. A summary and conclusions are given in Sec. $\mathrm{V}$.

\section{FORMALISM}

Our one-dimensional system involves two half-infinite leads connected by a central region where particles are scattered and is described by the Hamiltonian

$$
\hat{H}_{0}=\hat{H}_{\text {kin }}+\hat{V},
$$

where the potential $V(x)$ gives rise to single-particle scattering. Making use of the Lippmann-Schwinger (LS) scattering states $\left|\varphi_{\alpha k}\right\rangle$, we treat the scattering problem exactly; the index $\alpha=\mathrm{L} / \mathrm{R}$ distinguishes left/rightincoming states and we choose the wavevector $k>0$. The LS scattering states are eigenstates of the Hamiltonian $\hat{H}_{0}$,

$$
\left(\hat{H}_{\text {kin }}+\hat{V}\right)\left|\varphi_{\alpha k}\right\rangle=E_{k}\left|\varphi_{\alpha k}\right\rangle,
$$

with energy $E_{k}$. In the following, we will consider a spectrum linearized around $E_{\mathrm{F}}=\hbar \omega_{\mathrm{F}}$, i.e., $E_{k}=$ $E_{\mathrm{F}}+\hbar v_{\mathrm{F}}\left(k-k_{\mathrm{F}}\right)$ (this is a convenient simplification rather than a necessity). The asymptotics of the LS states $\left(\varphi_{\alpha k}(x)=\left\langle x \mid \varphi_{\alpha k}\right\rangle\right)$

$$
\begin{aligned}
& \varphi_{L k}(x)= \begin{cases}e^{i k x}+r_{L k} e^{i k d} e^{-i k x}, & x \rightarrow-\infty, \\
t_{k} e^{i k d} e^{i k x}, & x \rightarrow \infty,\end{cases} \\
& \varphi_{R k}(x)= \begin{cases}t_{k} e^{i k d} e^{-i k x}, & x \rightarrow-\infty, \\
e^{-i k x}+r_{R k} e^{i k d} e^{i k x}, & x \rightarrow \infty,\end{cases}
\end{aligned}
$$

is determined by the scattering matrix

$$
S_{k}=\left(\begin{array}{cc}
r_{L k} & t_{k} \\
t_{k} & r_{R k}
\end{array}\right)
$$

where $t_{L k}=t_{R k}=t_{k}$ in our time-reversal symmetric system. Different from more standard formulations, we have defined the scattering coefficients with additional phase factors $\exp (i k d)$ as this will simplify our expressions later. The Lippmann-Schwinger states satisfy the orthogonality

$$
\left\langle\varphi_{\alpha k} \mid \varphi_{\beta q}\right\rangle=2 \pi \delta(k-q) \delta_{\alpha \beta}
$$


and, in the absence of bound states as assumed here, the condition of completeness. The Hamiltonian $\hat{H}_{0}$ then can be rewritten in terms of the LS states as

$$
\hat{H}_{0}=\sum_{\alpha, \sigma} \int \frac{d k}{2 \pi} E_{k} \hat{c}_{\alpha k \sigma}^{\dagger} \hat{c}_{\alpha k \sigma}
$$

with the creation (annihilation) operator $\hat{c}_{\alpha k \sigma}^{\dagger}\left(\hat{c}_{\alpha k \sigma}\right)$ of LS scattering states with spin $\sigma$. With a voltage $V$ applied to the system, we assume a non-equilibrium steady state at an initial time $t_{0}\left(t_{0} \rightarrow-\infty\right)$ which we describe by the initial density matrix

$$
\hat{\rho}_{0}=\hat{\rho}\left(t_{0}\right)=\exp \left[-\beta \sum_{\alpha, \sigma} \int \frac{d k}{2 \pi}\left(E_{k}-\mu_{\alpha}\right) c_{\alpha k \sigma}^{\dagger} c_{\alpha k \sigma}\right]
$$

with the chemical potential $\mu_{L / R}=E_{\mathrm{F}} \pm \mathrm{eV} / 2$ in the left/right lead.

\section{A. Interaction matrix elements}

We consider an interaction between electrons described by the Hamiltonian

$$
\begin{aligned}
\hat{H}_{\mathrm{int}}=\frac{1}{2} \sum_{\sigma \sigma^{\prime}} \int d x & d x^{\prime} \hat{\psi}_{\sigma}^{\dagger}(x) \hat{\psi}_{\sigma^{\prime}}^{\dagger}\left(x^{\prime}\right) \\
& \times U\left(x, x^{\prime}\right) \hat{\psi}_{\sigma^{\prime}}\left(x^{\prime}\right) \hat{\psi}_{\sigma}(x)
\end{aligned}
$$

with $U\left(x, x^{\prime}\right)$ the interaction kernel. Here, we focus on a situation where the interaction can be assumed constant within the region of single-particle scattering. Typical physical systems we have in mind are quantum dots (with constant interaction inside the dot and vanishing outside) and quantum point contacts (where the interaction can be assumed large and constant between electrons in the quantum point contact region). Inspired by the capacitive form $Q^{2} / 2 C$ (with the charge $Q$ and capacitance $C$ ) we choose an electron-electron interaction kernel

$$
U\left(x, x^{\prime}\right)=U_{0} f(x) f\left(x^{\prime}\right)
$$

Using the Lippmann-Schwinger scattering states, we express the field operators as

$$
\hat{\psi}_{\sigma}(x)=\sum_{\alpha} \int \frac{d k}{2 \pi} \varphi_{\alpha k}(x) \hat{c}_{\alpha k \sigma}
$$

and can rewrite the Hamiltonian as

$$
\begin{aligned}
\hat{H}_{\mathrm{int}}= & \frac{U_{0}}{2} \sum_{\sigma \sigma^{\prime}} \sum_{\alpha \alpha^{\prime} \beta \beta^{\prime}} \int \frac{d k}{2 \pi} \frac{d k^{\prime}}{2 \pi} \frac{d q}{2 \pi} \frac{d q^{\prime}}{2 \pi} \\
& \times A_{\alpha^{\prime} k^{\prime}, \alpha k} A_{\beta^{\prime} q^{\prime}, \beta q} \hat{c}_{\alpha^{\prime} k^{\prime} \sigma^{\prime}}^{\dagger} \hat{c}_{\beta^{\prime} q^{\prime} \sigma^{\prime}}^{\dagger} \hat{c}_{\beta q \sigma^{\prime}} \hat{c}_{\alpha k \sigma}
\end{aligned}
$$

with the matrix elements

$$
A_{\alpha k, \beta q}=\int d x f(x) \varphi_{\alpha k}^{*}(x) \varphi_{\beta q}(x)
$$

Assuming a scattering potential $V(x)$ which vanishes outside the region $[-d / 2, d / 2]$, we consider a specific kernel with

$$
f(x)=\Theta(d / 2-|x|)
$$

allowing us to rewrite the matrix elements in Eq. (12) as

$$
A_{\alpha k, \beta q}=\left\langle\varphi_{\alpha k} \mid \varphi_{\beta q}\right\rangle-\int_{C_{d}} d x \varphi_{\alpha k}^{*}(x) \varphi_{\beta q}(x)
$$

with $C_{d}=\mathbb{R} \backslash[-d / 2, d / 2]$. The first term is given by the orthogonality condition Eq. (5) of the LS states, while the second term can be calculated using the asymptotic form Eq. (3) of the LS states. As a result, the overlap matrix elements can be expressed through the scattering coefficients alone,

$$
\begin{aligned}
& A_{\alpha k, \alpha q}=-i \frac{1-\left(r_{\alpha k}^{*} r_{\alpha q}+t_{\alpha k}^{*} t_{\alpha q}\right)}{k-q} e^{i(k-q) d / 2} \\
& A_{\alpha k, \beta q}=i \frac{\left(r_{\alpha k}^{*} t_{\beta q}+t_{\alpha k}^{*} r_{\beta q}\right)}{k-q} e^{i(k-q) d / 2}
\end{aligned}
$$

where we have dropped terms suppressed by the small parameter $1 /\left(d k_{\mathrm{F}}\right)$ and $\alpha \neq \beta$. For coinciding wave vectors $k=q$ the result reads (again, $\alpha \neq \beta$ )

$$
\begin{aligned}
& A_{\alpha k, \alpha k}=-i\left(r_{\alpha k}^{*} \partial_{k} r_{\alpha k}+t_{\alpha k}^{*} \partial_{k} t_{\alpha k}\right), \\
& A_{\alpha k, \beta k}=-i\left(r_{\alpha k}^{*} \partial_{k} t_{\beta k}+t_{\alpha k}^{*} \partial_{k} r_{\beta k}\right) .
\end{aligned}
$$

It is straightforward to generalize this scheme to situations where the kernel $f(x \in[-d / 2, d / 2])=1$ decays smoothly outside the (scattering) region $[-d / 2, d / 2]$. For the interacting resonant level mode $25 \mid 26$, the interaction can be described by the Hamiltonian Eq. (8) with a kernel $U\left(x, x^{\prime}\right)=U_{0} g(x) f\left(x^{\prime}\right)$ and the function $g(x)$ finite in the regions nearby but outside the dot; the interaction Hamiltonian Eq. (11) then can be expressed by the product of matrix elements $A_{\alpha k, \beta q}$ and a similar quantity with $f(x)$ replaced by $g(x)$ in Eq. (12). In the following, we concentrate on the capacitive interaction Eq. (9) with the specific choice Eq. 13.

Another situation where the knowledge of the scattering coefficients suffices to evaluate matrix elements is the interaction with long wave-length bosonic modes. As a specific example, we consider plasmonic excitations of a two-dimensional electron gas (2DEG) induced by scattering electrons, e.g., at a point contact; the interaction is described by the Hamiltonian 15 ,

$$
\hat{H}=\frac{1}{2 m} \sum_{\sigma} \int d x \hat{\psi}_{\sigma}^{\dagger}(x)\left[-i \hbar \partial_{x}-\frac{e}{c} \hat{A}(x)\right]^{2} \hat{\psi}_{\sigma}(x),
$$

where $\hat{A}(x)$ is the vector potential of the electromagnetic field induced by the two-dimensional plasmons. For the lowest transverse component of the plasmon wave function $\phi_{k}(x)$ one can write,

$$
\hat{A}(x)=\sum_{k} i k \gamma\left(\frac{2 \pi \hbar c^{2}}{\omega_{k}}\right)^{1 / 2}\left[\hat{b}_{k} \phi_{k}(x)-\hat{b}_{k}^{\dagger} \phi_{k}^{*}(x)\right],
$$


where $\hat{b}_{k}^{\dagger}\left(\hat{b}_{k}\right)$ are bosonic creation (annihilation) operators for the plasmon modes with wave vector $k$ and linear dispersion $\omega_{k}=v_{\mathrm{pl}} k$, with the plasmon velocity $v_{\mathrm{pl}}$ and a dimensionless geometrical factor $\gamma \sim 1$.

Typically, $v_{\mathrm{pl}} \gg v_{\mathrm{F}}$, and the conservation of energy $\delta E=v_{\mathrm{pl}} \hbar k_{\mathrm{pl}} \gg v_{\mathrm{F}} \hbar k$ implies that the plasmon wave length is much larger than the Fermi wave length, $\lambda_{\mathrm{pl}} \gg$ $\lambda_{\mathrm{F}}$. The size of the plasmon then exceeds, by several orders of magnitude, the scattering region (the size of the quantum point contact) and thus the interaction between the plasmons and electrons is dominated by the asymptotic scattering region where the electronic wave function has the form (3). Neglecting the spatial dependence of the plasmon wave function $\phi(x)=$ const., one again can express the interaction Hamiltonian (17) solely in terms of the non-interacting scattering matrix.

\section{B. Correction to Green's functions and scattering matrix}

We are interested in calculating the transport properties of a device, such as the mean current and the current-noise correlator. Within the Green's function approach, we need to know its single-particle version for the calculation of the current, while for the calculation of the current-current correlator, knowledge of the twoparticle Green's function is required. Below, we first derive the interaction correction to the single-particle and two-particle Green's functions, before focusing on the transport properties in the next section.

\section{Single-particle Green's function}

The single-particle Keldysh Green's functions ${ }^{32}$ are defined as

$$
\begin{aligned}
& G_{\nu \nu^{\prime}, \sigma}\left(x, t ; x^{\prime}, t^{\prime}\right) \\
& \quad=-i\left\langle\mathcal{T}_{K}\left[\hat{\psi}_{\sigma}\left(x, t_{\nu}\right) \hat{\psi}_{\sigma}^{\dagger}\left(x^{\prime}, t_{\nu^{\prime}}^{\prime}\right) \hat{S}_{K}\right]\right\rangle
\end{aligned}
$$

where the expectation value of an operator $\hat{A}$ is defined as $\langle\hat{A}\rangle=\operatorname{Tr}\left\{\hat{A} \hat{\rho}_{0}\right\}$ with the trace taken over all manyparticle states of the system and $\hat{\rho}_{0}$ is given by Eq. (7). We will skip the spin-index for the single-particle Green's functions in the following, as these are equal for both spin directions. The time-dependence of the field operator $\hat{\psi}(x, t)$ in the interaction representation is governed by the free Hamiltonian Eq. (6). $\nu\left(\nu^{\prime}\right)$ is a Keldysh index \pm telling on which branch of the Keldysh contour $\mathcal{C}=\left(t_{0}, \infty\right)_{+} \cup\left(\infty, t_{0}\right)_{-}$the time argument $t\left(t^{\prime}\right)$ is located (with $t_{0} \rightarrow-\infty$ ). $\mathcal{T}_{K}$ is the Keldysh time-ordering operator and $\hat{S}_{K}$ is the Keldysh evolution operator in the interaction representation.

The non-interacting Green's functions in frequency representation $G_{\nu \nu^{\prime}}^{(0)}\left(x, x^{\prime}, \omega\right)$ can be written in matrix form 32

$$
\begin{aligned}
\hat{G}^{(0)}\left(x, x^{\prime}, \omega\right)=\frac{-i}{v_{\mathrm{F}}} \sum_{\alpha} & \left(\varphi_{\alpha k_{\omega}}(x) \varphi_{\alpha k_{\omega}}^{*}\left(x^{\prime}\right) \hat{M}_{\alpha \omega}\right. \\
& \left.+\int \frac{d k}{2 \pi i} \frac{\varphi_{\alpha k}(x) \varphi_{\alpha k}^{*}\left(x^{\prime}\right)}{k-k_{\omega}-i \delta} \hat{\sigma}_{z}\right)
\end{aligned}
$$

with the wavevector $k_{\omega}=k_{\mathrm{F}}+\left(\omega-\omega_{\mathrm{F}}\right) / v_{\mathrm{F}}$, the matrix

$$
\hat{M}_{\alpha \omega}=\left(\begin{array}{cc}
-n_{\alpha}(\omega) & -n_{\alpha}(\omega) \\
1-n_{\alpha}(\omega) & 1-n_{\alpha}(\omega)
\end{array}\right)
$$

and the Pauli matrix $\hat{\sigma}_{z}$. Here, $n_{L / R}(\omega)$ is the Fermi distribution for the left/right lead. The integral in the expression Eq. 20 of the non-interacting Green's functions can be explicitly calculated for coordinates outside of the scattering region, i.e., $|x|,\left|x^{\prime}\right|>d / 2$, by inserting the asymptotic wave functions Eq. (3). E.g., for $x>x^{\prime}>d / 2$, we obtain

$$
\begin{aligned}
\hat{G}^{(0)}\left(x, x^{\prime}, \omega\right)= & \frac{-i}{v_{\mathrm{F}}} e^{i k_{\omega}\left(x-x^{\prime}\right)}\left(\hat{M}_{L \omega}+\hat{\sigma}_{z}\right) \\
& +\frac{-i}{v_{\mathrm{F}}} e^{-i k_{\omega}\left(x-x^{\prime}\right)} \hat{M}_{R \omega} \\
& +\frac{-i}{v_{\mathrm{F}}} R_{k_{\omega}} e^{i k_{\omega}\left(x-x^{\prime}\right)}\left(\hat{M}_{R \omega}-\hat{M}_{L \omega}\right) \\
& +\frac{-i}{v_{\mathrm{F}}} r_{R k_{\omega}} e^{i k_{\omega}\left(x+x^{\prime}-d\right)}\left(\hat{M}_{R \omega}+\hat{\sigma}_{z}\right) \\
& +\frac{-i}{v_{\mathrm{F}}} r_{R k_{\omega}}^{*} e^{-i k_{\omega}\left(x+x^{\prime}-d\right)} \hat{M}_{R \omega}
\end{aligned}
$$

where $R_{k_{\omega}}=\left|r_{R k_{\omega}}\right|^{2}$ and $T_{k_{\omega}}=\left|t_{L k_{\omega}}\right|^{2}$ and we have used $T_{k_{\omega}}+R_{k_{\omega}}=1$ to simplify the expression. Eq. 22 expresses the non-interacting Green's functions outside the scattering region in terms of scattering matrix coefficients; as we will see below, the (lowest order) corrections due to interaction assume the same structure and can be formulated in terms of a renormalization of the scattering coefficients.
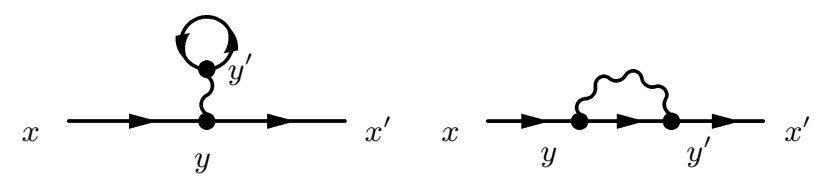

FIG. 1: The Hartree and Fock diagrams give rise to the first order corrections to the Green's functions given by Eq. 23. and 24 .

The first-order correction $\hat{G}^{(1)}$ to the single-particle Green's function is given by the first-order expansion of Eq. (19) in the interaction Hamiltonian. There are two contributions in lowest-order, a Hartree and a Fock term, cf. Fig. 1, for which we find the expressions 


$$
\begin{aligned}
& \hat{G}^{(1 \mathrm{H})}\left(x, x^{\prime}, \omega\right)=-\frac{2 i}{\hbar} \int d y d y^{\prime} U\left(y, y^{\prime}\right) \int \frac{d \tilde{\omega}}{2 \pi} \hat{G}^{(0)}(x, y, \omega) \hat{\sigma}_{z} G_{+-}^{(0)}\left(y^{\prime}, y^{\prime}, \tilde{\omega}\right) \hat{G}^{(0)}\left(y, x^{\prime}, \omega\right), \\
& \hat{G}^{(1 \mathrm{~F})}\left(x, x^{\prime}, \omega\right)=\frac{i}{\hbar} \int d y d y^{\prime} U\left(y, y^{\prime}\right) \int \frac{d \tilde{\omega}}{2 \pi} \hat{G}^{(0)}(x, y, \omega) \hat{\sigma}_{z} G_{+-}^{(0)}\left(y, y^{\prime}, \tilde{\omega}\right) \hat{G}^{(0)}\left(y^{\prime}, x^{\prime}, \omega\right) .
\end{aligned}
$$

The real-space non-interacting Green's functions $\hat{G}^{(0)}\left(x, x^{\prime}, \omega\right)$ can be expressed through the scattering states using

$$
\hat{G}^{(0)}\left(x, x^{\prime}, \omega\right)=\sum_{\alpha} \int \frac{d k}{2 \pi} \hat{g}_{\alpha k}^{(0)}(\omega) \varphi_{\alpha k}(x) \varphi_{\alpha k}^{*}\left(x^{\prime}\right),
$$

where $\hat{g}_{\alpha k}^{(0)}(\omega)$ are the non-interacting Green's functions (in $k$-space)

$$
\hat{g}_{\alpha k}^{(0)}(\omega)=g_{k}^{R}(\omega)\left(\hat{M}_{\alpha \omega}+\hat{\sigma}_{z}\right)-g_{k}^{A}(\omega) \hat{M}_{\alpha \omega}
$$

with the retarded/advanced Green's functions $g_{k}^{R / A}(\omega)=$ $\left(\omega-\omega_{k} \pm i \delta\right)^{-1}$ and $\omega_{k}=E_{k} / \hbar$. For our kernel Eq. (9) and the specific choice of $f(x)$ in Eq. (13), the integrations over the internal coordinates reduce to integrations of two scattering states within the region $[-d / 2, d / 2]$. We replace these integrals by integrations over the asymptotic regions as in Sec. IIA before in order to avoid the integration over the scattering region. As a result, these overlaps can be expressed through the matrix elements $A_{\alpha k, \beta q}$ defined in Eq. (15). The expressions for the Hartree and Fock corrections can be evaluated for all coordinates outside of the scattering region. Comparing for each choice of coordinate positions (to the left/right of the scatterer) the corrections with the non-interacting Green's function itself, we recognize that we can cast all effects of interaction into a renormalization of the scattering coefficients. E.g., for $x, x^{\prime}>d / 2$, the corrections assume the form of the three last terms in Eq. (22) and we can define the renormalization $r_{R k}^{(1)}$ to the reflection coefficient $r_{R k}$ in a consistent manner such as to capture all three corrections. The corrections to the scattering matrix again involve Hartree- and Fock contributions, $t_{\alpha k}^{(1)}=t_{\alpha k}^{(1 \mathrm{H})}+t_{\alpha k}^{(1 \mathrm{~F})}$ and $r_{\alpha k}^{(1)}=r_{\alpha k}^{(1 \mathrm{H})}+r_{\alpha k}^{(1 \mathrm{~F})}$; the Hartree terms are given by

$$
\begin{array}{r}
t_{L k}^{(1 \mathrm{H})}=-\frac{2 U_{0}}{\hbar v_{\mathrm{F}}} \sum_{\beta} \int \frac{d q}{2 \pi} n_{\beta q}\left(i t_{k} A_{L k, L k} A_{\beta q, \beta q}\right. \\
\left.+i r_{R k} A_{R k, L k} A_{\beta q, \beta q}\right) \\
r_{R k}^{(1 \mathrm{H})}=-\frac{2 U_{0}}{\hbar v_{\mathrm{F}}} \sum_{\beta} \int \frac{d q}{2 \pi} n_{\beta q}\left(i r_{R k} A_{R k, R k} A_{\beta q, \beta q}\right. \\
\left.+i t_{k} A_{L k, R k} A_{\beta q, \beta q}\right)
\end{array}
$$

and the corresponding corrections $t_{R k}^{(1 \mathrm{H})}$ and $r_{L k}^{(1 \mathrm{H})}$ are obtained by interchanging $\mathrm{R} \leftrightarrow \mathrm{L}$. The $q$ - and $k$-dependent parts in the above expressions factorize and we find the simpler results

$$
\begin{aligned}
& t_{\alpha k}^{(1 \mathrm{H})}=-\frac{U_{0} N_{\mathrm{int}}}{\hbar v_{\mathrm{F}}} \partial_{k} t_{k}, \\
& r_{\alpha k}^{(1 \mathrm{H})}=-\frac{U_{0} N_{\mathrm{int}}}{\hbar v_{\mathrm{F}}} \partial_{k} r_{\alpha k},
\end{aligned}
$$

with the particle number $N_{\text {int }}$ in the interaction region given by

$$
\begin{aligned}
N_{\mathrm{int}} & =\sum_{\sigma} \int_{-d / 2}^{d / 2} d y\left\langle\hat{\psi}_{\sigma}^{\dagger}(y, t) \hat{\psi}_{\sigma}(y, t)\right\rangle \\
& =2 \int \frac{d q}{2 \pi}\left(n_{L q} A_{L q, L q}+n_{R q} A_{R q, R q}\right),
\end{aligned}
$$

where $n_{\alpha k}=n_{\alpha}\left(E_{k}\right)$ and the factor 2 accounts for the two spin directions (note that the $k$-dependent factors in Eq. (27) reduce to a derivative of the scattering amplitudes, i.e., the brackets in Eq. 277 reduce to $A_{\beta q, \beta q} \partial_{k} t_{k}$ and $A_{\beta q, \beta q} \partial_{k} r_{R k}$, respectively).

Given the corrections to the scattering amplitudes, we can calculate the corrections to the transmission and reflection probabilities $T_{k}^{(1 \mathrm{H})}$ and $R_{k}^{(1 \mathrm{H})}=-T_{k}^{(1 \mathrm{H})}$,

$$
T_{k}^{(1 \mathrm{H})}=-\frac{U_{0} N_{\mathrm{int}}}{\hbar v_{\mathrm{F}}} \partial_{k} T_{k} .
$$

The result is easily interpreted: the Hartree interaction effectively shifts the scattering potential with respect to the energy of the incoming particle and thus the transmission characteristics of the scatterer is shifted accordingly; Eq. (30) then describes the lowest order correction of $T_{k}$ due to a shift in wavelength by $\delta k=-U_{0} N_{\text {int }} / \hbar v_{\mathrm{F}}$.

The Fock correction to the scattering matrix assumes the form

$$
\begin{aligned}
& t_{L k}^{(1 \mathrm{~F})}=\frac{U_{0}}{\hbar v_{\mathrm{F}}} \sum_{\beta} \int \frac{d q}{2 \pi} n_{\beta q}\left(i t_{k} A_{L k, \beta q} A_{\beta q, L k}\right. \\
&\left.+i r_{R k} A_{R k, \beta q} A_{\beta q, L k}\right), \\
& r_{R k}^{(1 \mathrm{~F})}=\frac{U_{0}}{\hbar v_{\mathrm{F}}} \sum_{\beta} \int \frac{d q}{2 \pi} n_{\beta q}(\left(i r_{R k} A_{R k, \beta q} A_{\beta q, R k}\right. \\
&\left.+i t_{k} A_{L k, \beta q} A_{\beta q, R k}\right) .
\end{aligned}
$$

The corrections $t_{R k}^{(1 \mathrm{~F})}$ and $r_{L k}^{(1 \mathrm{~F})}$ can be found by exchanging $\mathrm{R} \leftrightarrow \mathrm{L}$ in the expressions above. While for the Hartree term the corrections to the transmission coefficients are equal, i.e., $t_{R k}^{(1 \mathrm{H})}=t_{L k}^{(1 \mathrm{H})}$, this is in general not the case for 
the Fock contribution (however, equality holds in equilibrium). The corrections to the transmission and reflection probabilities $T_{k}^{(1 \mathrm{~F})}=-R_{k}^{(1 \mathrm{~F})}$ are

$$
\begin{aligned}
T_{k}^{(1 \mathrm{~F})} & =-\frac{U_{0}}{\hbar v_{\mathrm{F}}} \sum_{\beta} \int \frac{d q}{2 \pi} \frac{n_{\beta q}}{(k-q)^{2}} \\
& \times\left[i r_{\beta k} t_{k}^{*}\left(t_{k} r_{\beta q}^{*}+t_{q} r_{\beta k}^{*}-t_{q} r_{\beta q}^{*}\right)+\text { c.c. }\right] .
\end{aligned}
$$

The modifications to the current and the current-current correlator due to the renormalization of the singleparticle Green's functions will be discussed in section III below. Higher order perturbation theory might generate terms of different form which cannot be incorporated within a simple renormalization of the scattering coefficients.

\section{Two-particle Green's function}

The two-particle Keldysh Green's functions are defined as

$$
\begin{aligned}
& \mathcal{G}_{\sigma \pi}\left(1,2 ; 1^{\prime}, 2^{\prime}\right) \\
& \quad=(-i)^{2}\left\langle\mathcal{T}_{K}\left(\hat{\psi}_{\sigma}(1) \hat{\psi}_{\pi}(2) \hat{\psi}_{\pi}^{\dagger}\left(2^{\prime}\right) \hat{\psi}_{\sigma}^{\dagger}\left(1^{\prime}\right) \hat{S}_{K}\right)\right\rangle,
\end{aligned}
$$

where the variables $i=1,2,1^{\prime}, 2^{\prime}$ represent both space and time coordinates as well as the Keldysh index, i.e., $i=\left(x_{i}, t_{i}, \nu_{i}\right)$. The two-particle Green's functions can be split into a reducible and an irreducible part, $\mathcal{G}_{\sigma \pi}=$ $\mathcal{G}_{\sigma \pi}^{(\mathrm{red})}+\mathcal{G}_{\sigma \pi}^{(\mathrm{irr})}$, where the reducible part can be expressed through single-particle Green's functions as

$$
\begin{aligned}
\mathcal{G}_{\sigma \pi}^{(\mathrm{red})}\left(1,2 ; 1^{\prime}, 2^{\prime}\right)=G & \left(1,1^{\prime}\right) G\left(2,2^{\prime}\right) \\
& -\delta_{\sigma \pi} G\left(1,2^{\prime}\right) G\left(2,1^{\prime}\right)
\end{aligned}
$$

with the single-particle Green's function $G\left(1,1^{\prime}\right)=$ $G_{\nu_{1} \nu_{1}^{\prime}}\left(x_{1}, t_{1}, x_{1}^{\prime}, t_{1}^{\prime}\right)$, cf. Eq. (19), and similar for the other coordinates (again, we ignore the spin index). The non-interacting reducible two-particle Green's functions $\left(\mathcal{G}_{\sigma \pi}^{(\text {red })}\right)^{(0)}$ and corrections due to interaction (e.g., $\left.\left(\mathcal{G}_{\sigma \pi}^{(\text {red })}\right)^{(1)}\right)$ can be calculated from the non-interacting single-particle Green's functions $\hat{G}^{(0)}$ and the correction $\hat{G}^{(1)}$ to the single-particle Green's function.
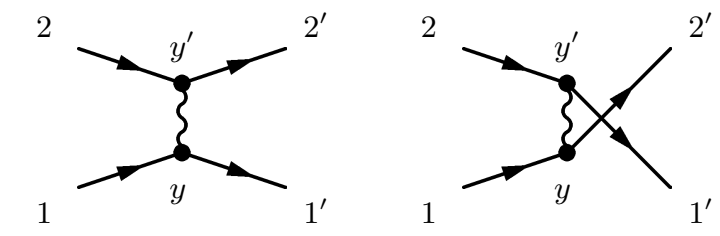

FIG. 2: There are two processes contributing to the correction to the irreducible two-particle Green's function in first order given by Eq. (35). While the second process only contributes for equal spins, the first one also gives a contribution for opposite spins.

The remaining irreducible part $\mathcal{G}_{\sigma \pi}^{(\mathrm{irr})}$ cannot be expressed through single-particle Green's functions and is given by the connected diagrams of Eq. (33). The lowestorder contribution to the irreducible two-particle Green's function involves two contributions, cf. Fig. 2, and can be evaluated for coordinates outside the scattering region and arbitrary Keldysh indices using the non-interacting Green's function in Eq. 20); to first order in the interaction (note that $\left(\mathcal{G}_{\sigma \pi}^{\text {(irr) }}\right)^{(\sigma)}=0$ ), we obtain in frequency representation with $\tilde{i}=\left(x_{i}, \omega_{i}, \nu_{i}\right)$,

$$
\begin{aligned}
\left(\mathcal{G}_{\sigma \pi}^{(\mathrm{irr})}\right)^{(1)}\left(\tilde{1}, \tilde{2} ; \tilde{1}^{\prime}, \tilde{2}^{\prime}\right)=i \frac{U_{0}}{\hbar} 2 & \delta\left(\omega_{1}+\omega_{2}-\omega_{1}^{\prime}-\omega_{2}^{\prime}\right) \int_{-d / 2}^{d / 2} d y d y^{\prime} \\
\times \sum_{\gamma} \gamma[ & G_{\nu_{1} \gamma}^{(0)}\left(x_{1}, y, \omega_{1}\right) G_{\nu_{2} \gamma}^{(0)}\left(x_{2}, y^{\prime}, \omega_{2}\right) G_{\gamma \nu_{2^{\prime}}}^{(0)}\left(y^{\prime}, x_{2}^{\prime}, \omega_{2}^{\prime}\right) G_{\gamma \nu_{1}}^{(0)}\left(y, x_{1}^{\prime}, \omega_{1}^{\prime}\right) \\
& \left.-\delta_{\sigma \pi} G_{\nu_{1} \gamma}^{(0)}\left(x_{1}, y, \omega_{1}\right) G_{\nu_{2} \gamma}^{(0)}\left(x_{2}, y^{\prime}, \omega_{2}\right) G_{\gamma \nu_{2^{\prime}}}^{(0)}\left(y, x_{2}^{\prime}, \omega_{2}^{\prime}\right) G_{\gamma \nu_{1}^{\prime}}^{(0)}\left(y^{\prime}, x_{1}^{\prime}, \omega_{1}^{\prime}\right)\right] .
\end{aligned}
$$

The integrals over the region $[-d / 2, d / 2]$ are calculated in the same way as in Sec. II A above.

\section{CORRECTION TO CURRENT AND NOISE}

Next, we determine the interaction corrections to the current and current-current correlator as calculated from single-particle and two-particle Green's functions; the irreducible contributions to the latter will give rise to spin correlations in an asymmetric device. 


\section{A. Current}

The current $j(x, t)$ derives from the single-particle Green's function via

$$
j(x, t)=2 \hat{\mathcal{D}}_{x} G_{+-}\left(x, t, x^{\prime}, t\right),
$$

where $\hat{\mathcal{D}}_{x}=(e \hbar / 2 m)\left(\partial_{x^{\prime}}-\partial_{x}\right)$ and the limit $x^{\prime} \rightarrow x$ is taken in the end. The factor 2 accounts for the two spin orientations of the electrons. We determine the current to the right of the scattering and interaction region, $x>d / 2$. As the effect of interactions on the singleparticle Green's function can be expressed through a renormalization of the scattering coefficients, the same applies to the current and we arrive at the well-known Landauer formula

$$
j^{(0)}+j^{(1)}=2 e v_{\mathrm{F}} \int \frac{d k}{2 \pi}\left[T_{k}+T_{k}^{(1)}\right]\left(n_{L k}-n_{R k}\right)
$$

with $T_{k}^{(1)}=T_{k}^{(1 \mathrm{H})}+T_{k}^{(1 \mathrm{~F})}$ given in Eqs. 30 and 32 .

\section{B. Current-current correlator}

Let us now turn our attention to the current-current correlator

$$
\begin{aligned}
& S_{\sigma \pi}\left(\omega, x_{1}, x_{2}\right) \\
& \quad=\int d \tau\left\langle\left\langle\mathcal{T}_{K}\left(\hat{j}_{\sigma}\left(x_{1}, \tau_{-}\right) \hat{j}_{\pi}\left(x_{2}, 0_{+}\right) \hat{S}_{K}\right)\right\rangle\right\rangle e^{i \omega \tau}
\end{aligned}
$$

behind the scatterer, $x_{1}, x_{2}>d / 2$. Below, we focus on the zero-frequency correlator which is independent of $x_{1}$ and $x_{2}$, i.e., $S_{\sigma \pi}(0)=S_{\sigma \pi}\left(0, x_{1}, x_{2}\right)$, as explained in Ref. 33. The current-current correlator can be expressed in terms of two-particle Green's functions and thus can be split into reducible and irreducible parts, $S_{\sigma \pi}=S_{\sigma \pi}^{(\text {red })}+S_{\sigma \pi}^{(\text {irr })}$. The reducible part vanishes for opposite spins $\sigma \neq \pi$, while for equal spins it is expressible in terms of single-particle Green's functions,

$$
S_{\sigma \sigma}^{\text {(red) }}(0)=-\hat{\mathcal{D}}_{x_{1}} \hat{\mathcal{D}}_{x_{2}} \int \frac{d \omega}{2 \pi} G_{-+}\left(x_{1}, x_{2}^{\prime}, \omega\right) G_{+-}\left(x_{2}, x_{1}^{\prime}, \omega\right) .
$$

The irreducible part contributies to both, equal and opposite spins, and can be expressed as

$$
S_{\sigma \pi}^{(\mathrm{irr})}(0)=\hat{\mathcal{D}}_{x_{1}} \hat{\mathcal{D}}_{x_{2}} \int \frac{d \omega_{1} d \omega_{2}}{(2 \pi)^{2}} \mathcal{G}_{\sigma \pi}^{\text {(irr) }}\left(1_{-}, 2_{+}, 1_{-}^{\prime}, 2_{+}^{\prime}\right),
$$

where we explicitely indicated the Keldysh indices and $\omega_{1^{\prime}, 2^{\prime}}=\omega_{1,2}$.

In the non-interacting case, the irreducible Green's function vanishes and the current-current correlator is given by the well-known expression $\frac{415}{4}$

$$
\begin{aligned}
S_{\sigma \pi}^{(0)}(0)= & \delta_{\sigma \pi} e^{2} v_{\mathrm{F}} \int \frac{d k}{2 \pi} \\
\times & {\left[T_{k}^{2}\left(n_{L k}\left(1-n_{L k}\right)+n_{R k}\left(1-n_{R k}\right)\right)\right.} \\
& \left.+R_{k} T_{k}\left(n_{L k}\left(1-n_{R k}\right)+n_{R k}\left(1-n_{L k}\right)\right)\right] .
\end{aligned}
$$

There are two kinds of (first-order) interaction corrections to the noise correlator: those due to the reducible part $S_{\sigma \pi}^{\text {(red) }}$ can be expressed through the renormalization of the transmission probability $T_{k}$ in Eq. (41). The contribution to the irreducible part $S_{\sigma \pi}^{(\mathrm{irr})}$ gives rise to additional correlations which go beyond a simple renormalization.

\section{Reducible corrections}

The expansion of the reducible part Eq. 39 in the interaction produces corrections originating from those in the single-particle Green's functions and we find

$$
\begin{aligned}
& S_{\sigma \pi}^{(\mathrm{red})(1)}(0)=\delta_{\sigma \pi} e^{2} v_{\mathrm{F}} \int \frac{d k}{2 \pi} \\
& \times\left[2 T_{k} T_{k}^{(1)}\left(n_{L k}\left(1-n_{L k}\right)+n_{R k}\left(1-n_{R k}\right)\right)\right. \\
& \left.\quad+\left(1-2 T_{k}\right) T_{k}^{(1)}\left(n_{L k}\left(1-n_{R k}\right)+n_{R k}\left(1-n_{L k}\right)\right)\right]
\end{aligned}
$$

which corresponds to the non-interacting expression Eq. (41) with a renormalized transmission probability $T_{k}+T_{k}^{(1)}$ and expanded to first order in $T_{k}^{(1)}$.

\section{Irreducible corrections}

Restricting ourselves to zero-temperature $(\vartheta=0)$, we find the first-order contribution to the irreducible twoparticle Green's function Eq. (35) with all coordinates to the right of the barrier $x_{i}, x_{i}^{\prime}>d / 2$ and evaluate the expression Eq. 40 for the irreducible noise correlator to arrive at

$$
\begin{gathered}
\left.S_{\sigma \pi}^{(\mathrm{irr})(1)}(0)\right|_{\vartheta=0}=-e^{2} \frac{U_{0}}{\hbar} \operatorname{sign}\left(\mu_{\mathrm{L}}-\mu_{\mathrm{R}}\right) \int_{k_{\min }}^{k_{\max }} \frac{d k_{1} d k_{2}}{(2 \pi)^{2}} \\
\times\left[\left(i r_{L k_{1}}^{*} t_{k_{1}} r_{L k_{2}}^{*} t_{k_{2}} A_{R k_{1}, L k_{1}} A_{R k_{2}, L k_{2}}\right.\right. \\
\left.\quad-i r_{R k_{1}}^{*} t_{k_{1}} r_{R k_{2}}^{*} t_{k_{2}} A_{L k_{1}, R k_{1}} A_{L k_{2}, R k_{2}}\right) \\
-\delta_{\sigma \pi}\left(i r_{L k_{1}}^{*} t_{k_{1}} r_{L k_{2}}^{*} t_{k_{2}} A_{R k_{1}, L k_{2}} A_{R k_{2}, L k_{1}}\right. \\
\left.\left.\quad-i r_{R k_{1}}^{*} t_{k_{1}} r_{R k_{2}}^{*} t_{k_{2}} A_{L k_{1}, R k_{2}} A_{L k_{2}, R k_{1}}\right)\right]
\end{gathered}
$$

where $k_{\max / \min }=\max / \min \left(k_{\mathrm{FL}} / k_{\mathrm{FR}}\right)$. The above correction vanishes for the symmetric setup where $r_{L k}=r_{R k}$ and $A_{R k, L q}=A_{L k, R q}$; on the other hand, interactions combined with an asymmetric scatterer, a case that has not been addressed previously, generate a finite correlation already in lowest order in $U_{0}$.

In summary, for equal spins, the correlation (or noise), due to scattering and fermionic statistics already present without interaction, is modified due to interactions via the renormalization of the transmission (in the reducible 
(a)

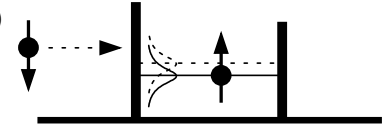

(b) $P_{L}$

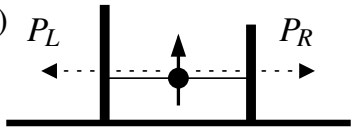

FIG. 3: (a) A (spin-up) electron on the dot shifts the resonance level for incoming (spin-down) electrons, thereby modulating the (spin-down) current across the scatterer. (b) For an asymmetric scatterer, the escape probability of the (spinup) electron to the left $\left(P_{L}\right)$ and right $\left(P_{R}\right)$ leads are different, generating a net (spin-up) current which is correlated with the (spin-down) current modulation.

contribution) and by the additional irreducible contribution given by Eq. (43) (note that this first-order contribution may vanish for equal spins due to the Pauli principle, as, e.g., for a single-level quantum dot). Opposite-spin correlations are absent in the non-interacting case, and a finite result is generated to first order in the interaction for an asymmetric scatterer by the first pair of terms in Eq. (43) and to second order in $U_{0}$ for a symmetric scatterer. We attribute the origin of the asymmetry-induced correlations to the following effect (we restrict the discussion to the case of an asymmetric quantum dot, cf. Fig. 3): First of all, we note that, by virtue of Pauli's exclusion principle, all interaction-induced modifications of the noise correlator are due to the presence of spin $\sigma$ particles on the dot modifying the current of spin $-\sigma$ particles traversing the dot. The finite correlation arises from the change in current $\delta I_{-\sigma}$ due to the presence of particles $\delta N_{\text {int } \sigma}$ on the dot, cf. Fig. 3 (a). For a symmetric scatterer, the decay of the charge $\delta N_{\mathrm{int} \sigma}$ to the unblocked scattering state is symmetric and the undirected current leads to a vanishing current-current correlator. On the other hand, in an asymmetric dot, the charge $\delta N_{\text {int } \sigma}$ decays in an asymmetric manner and its current $\delta I_{\sigma}$ is correlated with $\delta I_{-\sigma}$, cf. Fig. 3 (b).

\section{SINGLE RESONANCE LEVEL}

The above results, which apply to an arbitrary scatterer, can be simplified and further developed when choosing a particular scatterer. Here, we consider a simple example in the form of a scatterer with a single resonance level at energy $E_{\text {res }}=E_{\mathrm{F}}+\hbar v_{\mathrm{F}}\left(k_{\mathrm{res}}-k_{\mathrm{F}}\right)$, located at $x=0$ with vanishing extent $d \rightarrow 0$. This single-level quantum dot is described by the scattering matrix $S_{k}$ with amplitudes

$$
\begin{aligned}
t_{k} & =\frac{i \gamma}{\Delta k+i \gamma} \sqrt{1-\eta^{2}}, \\
r_{L k} & =\frac{\Delta k+i \eta \gamma}{\Delta k+i \gamma}, \\
r_{R k} & =\frac{\Delta k-i \eta \gamma}{\Delta k+i \gamma},
\end{aligned}
$$

where the wavevector $\Delta k=k-k_{\text {res }}$ is measured relative to the resonance level; the level width $\hbar v_{\mathrm{F}} \gamma$ is (a)

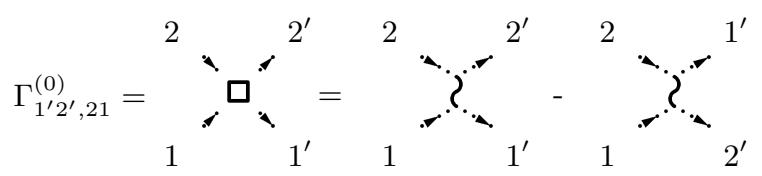

(b)

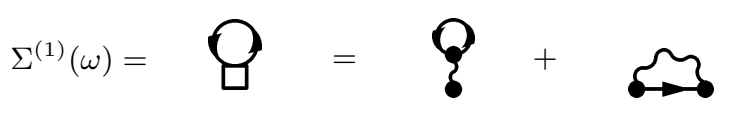

FIG. 4: (a) The two-particle vertex $\Gamma_{1^{\prime} 2^{\prime}, 21}^{(0)}$ is antisymmetrized with respect to the two incoming legs and to the two outgoing legs. (b) The first-order self-energy $\Sigma^{(1)}(\omega)$ consists of a Hartree and a Fock contribution.

parametrized by $\gamma$ and the dimensionless parameter $\eta \in$ $[-1,1]$ characterizes the asymmetry of the system. This scattering matrix generates a transmission probability $T_{k}=\left|t_{k}\right|^{2}=\left(1-\eta^{2}\right) /\left(1+(\Delta k / \gamma)^{2}\right)$.

The scattering matrix of the single resonance level model gives rise to matrix elements $A_{\alpha k, \beta q}$ of a particularly simple form, i.e.,

$$
A_{\alpha k, \beta q}=a_{\alpha}^{*} \phi_{k}^{*} a_{\beta} \phi_{q}
$$

with $a_{L / R}= \pm i \sqrt{1 \mp \eta}$ and $\phi_{k}=\sqrt{\gamma} /(\Delta k+i \gamma)$. The interaction Hamiltonian Eq. (11) then factorizes in the momenta, with individual terms given by

$$
\begin{aligned}
\hat{h}_{\mathrm{int}}=\frac{U_{0}}{2} a_{\alpha_{1}^{\prime}}^{*} a_{\alpha_{2}^{\prime}}^{*} a_{\alpha_{2}} a_{\alpha_{1}} \phi_{k_{1}^{\prime}}^{*} \phi_{k_{2}^{\prime}}^{*} \phi_{k_{2}} \phi_{k_{1}} \\
\quad \times \delta_{\sigma_{1} \sigma_{1}^{\prime}} \delta_{\sigma_{2} \sigma_{2}^{\prime}} \hat{c}_{\alpha_{1}^{\prime} k_{1}^{\prime} \sigma_{1}^{\prime}}^{\dagger} \hat{c}_{\alpha_{2}^{\prime} k_{2}^{\prime} \sigma_{2}^{\prime}}^{\dagger} \hat{c}_{\alpha_{2} k_{2} \sigma_{2}} \hat{c}_{\alpha_{1} k_{1} \sigma_{1}},
\end{aligned}
$$

and integration, summation over all wave vectors, lead, and spin indices providing the full interaction Hamiltonian $H_{\text {int }}$. Introducing the anti-symmetrized two-particle vertex

$$
\Gamma_{1^{\prime} 2^{\prime}, 21}^{(0)}=U_{0} a_{\alpha_{1}^{\prime}}^{*} a_{\alpha_{2}^{\prime}}^{*} a_{\alpha_{2}} a_{\alpha_{1}} \phi_{k_{1}^{\prime}}^{*} \phi_{k_{2}^{\prime}}^{*} \phi_{k_{2}} \phi_{k_{1}} S_{\sigma_{1}^{\prime} \sigma_{2}^{\prime}, \sigma_{2} \sigma_{1}}
$$

with $i=1,2,1^{\prime}, 2^{\prime}$ representing the multi-index $\left(\alpha_{i}, k_{i}, \sigma_{i}\right)$ and

$$
S_{\sigma_{1}^{\prime} \sigma_{2}^{\prime}, \sigma_{2} \sigma_{1}}=\delta_{\sigma_{1}^{\prime} \sigma_{1}} \delta_{\sigma_{2}^{\prime} \sigma_{2}}-\delta_{\sigma_{1}^{\prime} \sigma_{2}} \delta_{\sigma_{2}^{\prime} \sigma_{1}}
$$

the individual terms in Eq. 46, assume the form

$$
\hat{h}_{\mathrm{int}}=\frac{1}{4} \Gamma_{1^{\prime} 2^{\prime}, 21}^{(0)} \hat{c}_{\alpha_{1}^{\prime} k_{1}^{\prime} \sigma_{1}^{\prime}}^{\dagger} \hat{c}_{\alpha_{2}^{\prime} k_{2}^{\prime} \sigma_{2}^{\prime}}^{\dagger} \hat{c}_{\alpha_{2} k_{2} \sigma_{2}} \hat{c}_{\alpha_{1} k_{1} \sigma_{1}} .
$$

The Hartree and Fock digrams in Fig. 1 then collapse into one diagram, cf. Fig. 4 .

Given the simple structure of the vertex (47), we can easily sum the Hartree-Fock diagrams for the singleparticle Green's function (cf. Fig. 4(b)) with the help of the Dyson equation

$$
\begin{aligned}
& \hat{G}_{\alpha k, \alpha^{\prime} k^{\prime}}^{\Sigma^{(1)}}(\omega)=\hat{G}_{\alpha k, \alpha^{\prime} k^{\prime}}^{(0)}(\omega) \\
& \quad+\sum_{\beta, \beta^{\prime}} \int \frac{d q}{2 \pi} \frac{d q^{\prime}}{2 \pi} \hat{G}_{\alpha k, \beta q}^{(0)}(\omega) \hat{\Sigma}_{\beta q, \beta^{\prime} q^{\prime}}^{(1)}(\omega) \hat{G}_{\beta^{\prime} q^{\prime}, \alpha^{\prime} k^{\prime}}^{\Sigma^{(1)}}(\omega) .
\end{aligned}
$$


The first-order (Hartree-Fock) contribution to the selfenergy $\hat{\Sigma}^{(1)}$ assumes the following form (we use the Keldysh matrix notation and suppress the spin-indices as the self-energy is $\propto \delta_{\sigma^{\prime} \sigma}$ )

$$
\begin{aligned}
\hat{\Sigma}_{\alpha_{1}^{\prime} k_{1}^{\prime}, \alpha_{1} k_{1}}^{(1)}(\omega) & =\frac{1}{\hbar} \sum_{\alpha_{2}, \sigma_{2}} \int \frac{d k_{2}}{2 \pi} \Gamma_{1^{\prime} 2,21} n_{\alpha_{2}}\left(k_{2}\right) \hat{\sigma}_{z} \\
& =\frac{\tilde{U}}{\hbar} a_{\alpha_{1}^{\prime}}^{*} \phi_{k_{1}^{\prime}}^{*} \phi_{k_{1}} a_{\alpha_{1}} \hat{\sigma}_{z},
\end{aligned}
$$

with $\tilde{U}=U_{0} N_{\text {int }} / 2$ and $N_{\text {int }}$, cf. Eq. 29 , is the number of electrons on the level,

$$
N_{\mathrm{int}}=\int \frac{d(q / \gamma)}{2 \pi} \frac{(1-\eta) n_{L q}+(1+\eta) n_{R q}}{1+(\Delta q / \gamma)^{2}} .
$$

The self-energy is due to the Hartree interaction between electrons of opposite spins; the Hartree and Fock interaction between equal electrons cancel each other.

The Dyson equation (50) involves the non-interacting Green's function Eq. 20 transformed to $k$-space which is diagonal in the momentum and lead indices, $\hat{G}_{\alpha k, \beta q}^{(0)}(\omega)=$ $2 \pi \delta(k-q) \delta_{\alpha \beta} \hat{g}_{\alpha k}^{(0)}(\omega)$ with $\hat{g}_{\alpha k}^{(0)}(\omega)$ given by Eq. 26 . We solve the Dyson equation 50 for $\hat{G}_{\alpha k, \alpha^{\prime} k^{\prime}}^{\Sigma(1)}(\omega)$ in $k$-space and transform the result back to real space. The real-space Green's function $\hat{G}^{\Sigma(1)}\left(x, x^{\prime}, \omega\right)$ then assumes the same form as for the non-interacting case (cf. $\hat{G}^{(0)}\left(x, x^{\prime}, \omega\right)$ as given by Eq. 20 ) but with renormalized scattering coefficients

$$
\begin{aligned}
& \tilde{t}_{\alpha k}=t_{\alpha\left(k-\tilde{U} / \hbar v_{F}\right)}, \\
& \tilde{r}_{\alpha k}=r_{\alpha\left(k-\tilde{U} / \hbar v_{F}\right)} .
\end{aligned}
$$

This renormalization of the scattering amplitudes then corresponds to a mean-field shift of the resonance by $\tilde{U} / \hbar v_{F}$; the general results Eqs. 27) and (31) correspond to the first order expansion in $U_{0}$ of this result. Higherorder terms in the self-energy may not preserve the structure of the non-interacting Green's function, in which case they cannot be cast into a simple renormalization of the scattering coefficients.

The single-particle Green's function calculated above provides us with the corrections to the current $j$ and the reducible current-current correlator $S_{\sigma \sigma}^{(\mathrm{red})}$, cf. Eqs. (36) and (39). The non-interacting results for these two quantities, Eqs. (37) and (41), are modified by substituting the transmission probability $T_{k}$ with the expression $\tilde{T}_{k}=T_{\left(k-\tilde{U} / \hbar v_{F}\right)}$ shifted in energy due to the interaction. For zero temperature $(\vartheta=0)$ and in the linear-voltage regime, we obtain

$$
\begin{aligned}
\left.j^{\Sigma^{(1)}}\right|_{\vartheta=0, V \rightarrow 0} & =\frac{2 e^{2}}{h} \tilde{T}_{k_{F}} V, \\
\left.S_{\sigma \sigma}^{(\mathrm{red}) \Sigma^{(1)}}\right|_{\vartheta=0, V \rightarrow 0} & =\frac{e^{2}}{h}\left(1-\tilde{T}_{k_{F}}\right) \tilde{T}_{k_{F}}|e V| .
\end{aligned}
$$

The irreducible current-current correlator $S_{\sigma \pi}^{(\mathrm{irr})}$ is determined by the two-particle Green's function. Here, we stay with the simple first-order correction Eq. 43, as a proper resummation involves more complex diagrams including vertex corrections. The irreducible contribution to the current-current correlator is limited to opposite spins (where the reducible part does not contribute) and we obtain the result

$$
\begin{aligned}
& \left.S_{\uparrow \downarrow}^{(1)}(0)\right|_{\vartheta=0}=e^{2} \frac{4 U_{0}}{\hbar} \eta\left(1-\eta^{2}\right)^{2} \operatorname{sign}\left(\mu_{\mathrm{L}}-\mu_{\mathrm{R}}\right) \\
& \quad \times \int_{k_{\min }}^{k_{\max }} \frac{d k_{1} d k_{2}}{(2 \pi)^{2}} \frac{1}{\gamma^{2}} \frac{\Delta k_{1} / \gamma}{\left(1+\left(\Delta k_{1} / \gamma\right)^{2}\right)^{2}\left(1+\left(\Delta k_{2} / \gamma\right)^{2}\right)^{2}},
\end{aligned}
$$

which for small voltage reduces to

$$
\begin{aligned}
&\left.S_{\uparrow \downarrow}^{(1)}(0)\right|_{\vartheta=0, V \rightarrow 0}=e^{2} \frac{4 U_{0}}{\hbar} \eta\left(1-\eta^{2}\right)^{2}\left(\frac{e V}{\hbar v_{\mathrm{F}} \gamma}\right)^{2} \operatorname{sign}(V) \\
& \times \frac{\Delta k_{\mathrm{F}} / \gamma}{\left(1+\left(\Delta k_{\mathrm{F}}\right)^{2}\right)^{4}}
\end{aligned}
$$

Its sign

$$
\operatorname{sign}\left(\left.S_{\uparrow \downarrow}^{(1)}(0)\right|_{\vartheta=0, V \rightarrow 0}\right)=\operatorname{sign}(\eta) \operatorname{sign}(V) \operatorname{sign}\left(\Delta k_{\mathrm{F}}\right)
$$

can be understood on the basis of the mechanism described at the end of Sec. III B 2. The result (56) provides the lowest order in $V$ and $U_{0}$ result for the equalspin noise correlator; the opposite-spin correlator is generated by the combination of interaction and asymmetry and is given by Eq. (57).

It is instructive to compare our work with the results of Gogolin and Komnik ${ }^{20}$. These authors calculated the logarithm of the generating function $\log \chi$ for the full counting statistics (FCS) in the Anderson impurity model to second order in the interaction parameter $U / \Gamma$ (with $\Gamma$ the width of the level), using a particular diagrammatic technique, in linear response $(V \rightarrow 0)$ with the following results: i) The FCS assumes the same form as for noninteracting electrons but with renormalized transmission probabilities. ii) In the non-linear regime (finite voltage $V)$, spin-pairing correlations are generated to order $V^{2}$ in bias and to order $(U / \Gamma)^{2}$ in interaction (pairing correlations have been found $\sqrt{34}$ as well for a dot described within a master equation approach valid at large interaction $U$ ). These results are obtained for a symmetric dot. In our work, we extend the analysis to an arbitrary scatterer with capacitive-like Coulomb interaction in the scattering region. Rather than FCS, we limit ourselves to the calculation of the first two moments (current and noise) of the FCS and to first order effects in interaction. We confirm the renormalization of the single-particle scattering matrix in the linear response regime. For finite voltage $V$, we find that pairing correlations for electrons with opposite spins are enhanced for an asymmetric scatterer, i.e., a scatterer with different reflection coefficients $r_{L k} \neq r_{R k}$ for electrons incident from the left (L) and from the right $(\mathrm{R})$ lead, as they appear already in the first order in interaction $U / \Gamma$. 


\section{CONCLUSION}

We have extended the scattering matrix approach to include effects of interaction; while no restrictions have been imposed on the scatterer, our formalism applies to the special case of an interaction which is constant over the scattering region. Under these conditions, the fully interacting Hamiltonian can be expressed through the non-interacting (Lippmann-Schwinger) scattering states and scattering coefficients, hence the scattering aspect of the problem is dealt with in an exact way. On the part of the interaction, no knowledge is required about the wave function within the interaction region. The approach can be extended to the case where scattered electrons interact with electromagnetic and crystal degrees of freedom, photons, plasmons, and phonons.

In our analysis above, we have considered a constant interaction kernel and one may ask, how well this choice approximates the situation when the kernel is shortranged. For the single resonance level discussed in Sec. IV] the shape of the interaction kernel is not relevant; the matrix element Eq. 45 derives from the expression Eq. 15 for the finite scatterer in the limit of vanishing extent $d$ and any interaction kernel is always longranged. For a finite dot of dimension $d$, the shape of the interaction kernel $U\left(x, x^{\prime}\right)$ becomes relevant when its width drops below $d$. Here, we discuss the situation with sharp resonances and wave functions close to those of an isolated dot. As long as only a single resonance is involved, the modifications can be captured by a simple renormalization of the interaction strength $U_{0}$, with the Hartree and Fock contributions of similar weight. The Hartree contribution remains rather robust when including other occupied resonances, with a uniform renormalization of the interaction strength over the resonances (for a narrow kernel, the contributions from the lowest resonances, where the width of the kernel resolves the structure of the wave functions, is modified). For the constant kernel, the inter-resonance Fock terms are strongly suppressed as compared to the Hartree term, a consequence of the approximate orthogonality of the wave functions; a narrow kernel then enhances the relative importance of these (actually small) inter-resonance Fock terms. Overall, changing the kernel, we expect small effects on the renormalization of the scattering coefficients, on the transport current, and on the reducible part of the zero-frequency noise. For these quantities, the relevant physics involves electrons passing through one resonance and interacting with all the electrons on occupied resonances, which can be roughly included into a renormalization of $U_{0}$. Similarly, a small effect is expected in the irreducible part of the zero-frequency noise as long as only one resonance is involved in the trans- port. However, at larger voltage, more than one resonance contributes to the irreducible noise correlator and the interaction between electrons passing through different resonances becomes important; in this case, the specific enhancement of the inter-resonance Fock terms should be accounted for, e.g., via the introduction of additional 'cross-capacitances'. For an open dot with wide resonances and a short-range kernel, the wave functions in the dot have to be found explicitly when calculating the overlap matrix elements (12), the number of parameters increases, and the results can no longer be expressed through the scattering coefficients alone in the simple way we have done above.

As a first application of our approach, we have determined the one- and two-particle Green's functions within first order perturbation theory in the interaction parameter $U_{0}$ for a general scatterer - an analysis to higher order is more easily carried out for special scatterers, e.g., a single resonance level where the interaction Hamiltonian factorizes in $k$-space. While the linear-response corrections to the one- and the reducible part of the two-particle Green's functions can be cast into a renormalization of the scattering coefficients, additional terms of different form show up in the irreducible part of the two-particle Green's function. In the non-linear regime at high voltage, corrections appear which are beyond renormalization of the scattering matrix. Similarly, the interactioninduced corrections to the current and reducible part of the noise are accounted for through (the same) renormalized scattering coefficients. In addition, the interaction generates an irreducible contribution to the noise correlator which goes beyond renormalization of scattering corefficients. The latter gives rise to opposite-spin current correlations already to first order in $U_{0}$ for the case of an asymmetric scatterer, an effect which has not been addressed so far.

Our results are in agreement with those of Gogolin and Komnik $^{20}$ who reported a similar renormalization (in linear response and to second order in $U_{0}$ ) of scattering coefficients in their study of the full counting statistics of the (symmetric) Anderson model; their spin-pair correlation appears only in second order of the interaction as their scatterer is symmetric. While our study is first order in $U_{0}$, it applies to any scatterer with arbitrary scattering coefficients. We hope, that the simplifications which come with our approach can be used to generate new (as with the asymmetric scatterer) or more precise (as with the resummation of a class of diagrams for the single resonance level) results in other situations.

We thank Andreas Komnik for illuminating discussions and acknowledge financial support from the Swiss National Foundation, the Pauli Center for Theoretical Physics, and from the RFBR Grant \# 11-02-00744-a.

${ }^{1}$ R. Landauer, IBM J. Res. Dev. 1, 233 (1957).

${ }^{2}$ R. Landauer, Phil. Mag. 21, 863 (1970). 
3 M. Büttiker, Phys. Rev. Lett. 57, 1761 (1986).

${ }^{4}$ G.B. Lesovik, JETP Lett. 49, 592 (1989).

5 M. Büttiker, Phys. Rev. Lett. 65, 2901 (1990).

6 L.S. Levitov and G.B. Lesovik, JETP Lett. 58, 230 (1993).

7 L.S. Levitov, H. Lee and G.B. Lesovik, J. Math. Phys. 37, 4845 (1996).

8 Ya.M. Blanter and M. Büttiker, Phys. Rep. 336, 1 (2000).

${ }^{9}$ G.B. Lesovik and I.A. Sadovskyy, Phys. Usp. 54, 1007 (2011).

${ }^{10}$ L. Glazman and M. Pustilnik, in Nanophysics: Coherence and Transport, edited by H. Bouchiat et al. (Elsevier, Amsterdam, 2005), pp. 427-478.

11 K.J. Thomas et al., Phys. Rev. Lett. 77, 135 (1996).

12 I.V. Lerner, B.L. Altshuler, and Y. Gefen (eds.), Fundamental Problems of Mesoscopic Physics: Interactions and Decoherence, NATO Science Series II, Vol. 154 (Kluwer Academic Publishers, Dordrecht, 2004).

13 C. W. J. Beenakker and H. Schomerus, Phys. Rev. Lett. 86, 700 (2001).

14 C. W. J. Beenakker and H. Schomerus, Phys. Rev. Lett. 93, 096801 (2004).

15 A.V. Lebedev, G.B. Lesovik, and G. Blatter, Phys. Rev. B 81, 155421 (2010).

16 Y. Meir and N.S. Wingreen, Phys. Rev. Lett. 68, 2512 (1992).

17 P.W. Anderson, Phys. Rev. 124, 41 (1961).

18 R.M. Konik, H. Saleur, and A. Ludwig, Phys. Rev. B 66, 125304 (2002).

19 A. Schiller and S. Hershfield, Phys. Rev. B 58, 14978
(1998).

20 A.O. Gogolin and A. Komnik, Phys. Rev. B 73, 195301 (2006).

21 P. Mehta and N. Andrei, Phys. Rev. Lett. 96, 216802 (2006).

22 E. Boulat, H. Saleur, and P. Schmitteckert, Phys. Rev. Lett. 101, 140601 (2008).

23 A. Branschädel, E. Boulat, H. Saleur, and P. Schmitteckert, Phys. Rev. Lett. 105, 146805 (2010).

24 S.T. Carr, D.A. Bagrets, and P. Schmitteckert, Phys. Rev. Lett. 107, 206801 (2011).

25 P.B. Vigman and A.M. Finkel'shtein, Sov. Phys. JETP 48, 102 (1978).

26 P. Schlottmann, J. Magn. Magn. Mater. 7, 72 (1978).

27 D.S. Golubev, A.V. Galaktionov, and A.D. Zaikin, Phys. Rev. B 72, 205417 (2005).

28 M. Kindermann and Yu.V. Nazarov, Phys. Rev. Lett. 91, 136802 (2003); D.A. Bagrets and Yu.V. Nazarov, Phys. Rev. Lett. 94, 056801 (2005).

29 H. Ness, L. K. Dash, and R.W. Godby, Phys. Rev. B 82, 085426 (2010).

30 H. Ness and L. K. Dash, Phys. Rev. B 84, 235428 (2011).

31 G. Vignale and M. Di Ventra, Phys. Rev. B 79, 014201 (2009).

32 L.V. Keldysh, Sov. Phys. JETP 20, 1018 (1965).

33 G.B. Lesovik, condmat/0308137 (1999).

34 O. Sauret and D. Feinberg, Phys. Rev. Lett. 92, 106601 (2004). 